\title{
Molecular dynamics study of effect of wettability on rapid boiling over copper nanoparticle in water pool
}

\author{
Bo Zhang ${ }^{1}$ \\ ${ }^{1}$ Beijing Raymer Technology Co., Ltd. Beijing, 1000142, China
}

\begin{abstract}
Many special phenomena occur during rapid boiling of a liquid when it is largely superheated near the thermodynamic critical temperature which is much higher than the saturation temperature. However, although there are many experimental work reported the observation of rapid ejection near the surface region of the materials, they are limited in either length or time scales due to the limitation of classical macroscopic theory. In this work, molecular dynamics simulation of heat transfer from nanoparticle to a surrounding liquid pool is carried out to study the effect of solid-liquid interfacial wettability on the boiling phenomena of water around the nanoparticle. The interactions among copper atoms are described by the embedded atom method (EAM) potentials, and the TIP4P water model is used to describe water atoms. The results show that the interfacial thermal conductance is influenced by the interfacial wettability. Increasing the wettability between the particle and the fluid reduces the interfacial resistance. The nucleation of bubbles and formation of vapor occur more quickly for increased solid-liquid interfacial wettability. The results might change due to the uncertainties of the potential functions, therefore further investigation is worthwhile to study rapid boiling of water on nanoparticles using different water models.
\end{abstract}

\section{Introduction}

The concept of nanofluids was firstly proposed by Choi et al. [1] at Argonne National Laboratory in the United States in 1995. They proposed to suspend nanoscale metal or metal oxide nanoparticles into liquid base liquid in a certain way and proportion. For example, this can be a new type of heat transfer cooling refrigerant [2]. Nowadays nanofluid is a hot research topic all over the world. Many experimental results show that the thermal conductivity of nanofluids can be improved to a certain degree relative to that of its base fluid and hence has broad application potentials.

Molecular Dynamics (MD) simulation is an important method to study nanofluids. It was widely applied to nanofluids to explore the heat transfer mechanisms [3]. Many studies focused on the heat transfer enhancement of nanofluids in terms of experiments macroscopically, including the influence of different nanoparticles and different volume fraction on heat transfer [4]. Some use the MD method to study the problem of rapid heat exchange. The contact angles and density distributions of argon [5-6] and water droplets [7] on solid wall were obtained. The heterogeneous nucleation [8] of bubbles on solid wall and the molecular morphology of the adherent bubbles were observed. Yan et al. [9] studied the influence of different wall temperatures on the liquid film thickness in the near wall. Carey et al. [10] studied the effect of solid wall on the boiling process and found that the pressure and critical superheat of liquid molecules near the wall are significantly increased under the effect of solid wall, resulting in a certain liquid from the solid wall to reach the nucleation condition first to start boiling. Wang et al. [11] used platinum heating surfaces to simulate the thermal contact resistance of solid-liquid sections in a triangular cross-section nanoscale channel. Xue et al. [12] found that the strength of the contact resistance has a significant impact on the interaction between solid-liquid atoms.

Although many research on boiling nucleation has been conducted, the research on the influence of different solid wall properties on the nucleation phenomenon and the heat transfer mechanism lacks comprehensive understanding. Under different wall conditions, the interaction between the wall and the fluid atoms requires the simulation of large number of atoms to obtain more accurate macro information. With the reduction of the scale of the research object, the influence of the interfacial thermal resistance on the boiling nucleation cannot be neglected. In this paper, molecular dynamics simulations of near-surface cell-to-cell boiling system are established to simulate the rapid heat transfer process in the near wall under the condition of pool boiling. The effect of solid wall wettability on the liquid boiling is analyzed for the variation of water temperature distribution and number density of water molecules at different distances.

\section{Modelling}

The whole molecular system is consisted of a solid copper nanoparticle at the center of a water pool, as seen in Fig. 1. The simulation is performed by the Largescale 
Atomic/Molecular Massively Parallel Simulator (LAMMPS) under the framework of classical MD [13]. The visual graphs of system configuration and atomic motion are obtained by using the Visual Molecular Dynamics (VMD) and the Open Visualization Tool (OVITO) software. The system uses face-centered cubic
(FCC) unit cells to model the copper nanoparticle and the working fluid. Periodic boundary condition is applied in the $\mathrm{x}-, \mathrm{y}$ - and $\mathrm{z}$-directions. The total length (in the $\mathrm{x}$ direction), width (in the y-direction) and height (in the $\mathrm{z}$ direction) of the box are set as 10 nanometers.
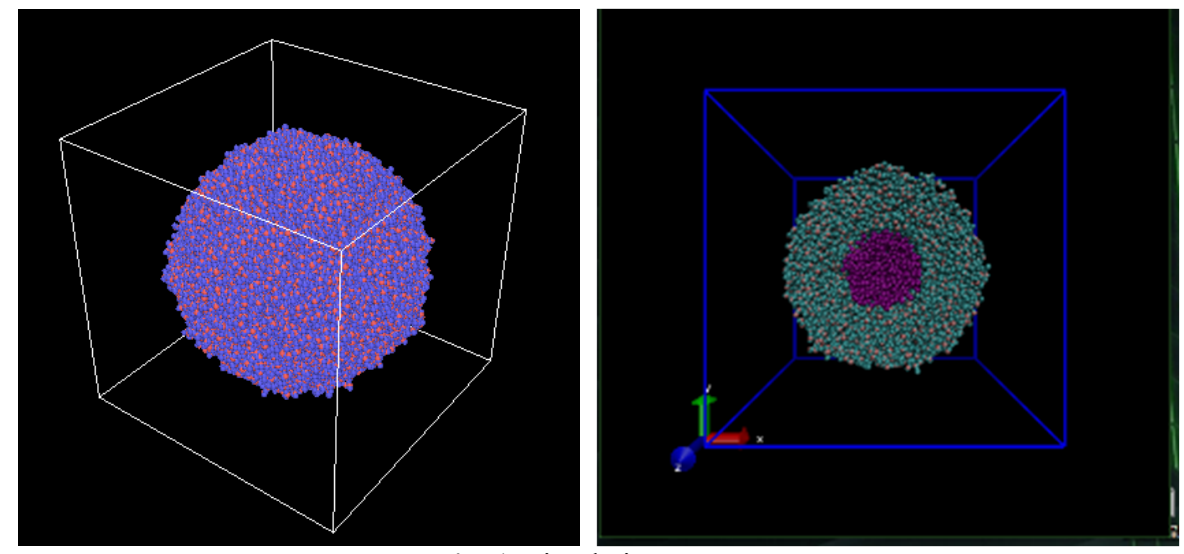

Fig. 1. Simulation system.

For copper nanoparticle, the lattice constant of FCC unit cell is set as $3.6149 \AA$, which enables the conversion of copper density as $8.96 \mathrm{~g} / \mathrm{cm} 3$. The copper nanoparticle is placed in the center of the water pool. It is divided into the fixed part and the heat source part. Copper atoms in the fixed part at the center region of the nanoparticle are fixed without atomic vibration, which could prevent the copper atoms from escaping in the whole simulation. The heat source part is adjacent to the fixed part, which is controlled by the Berendsen thermostats for heat flowing from the hot copper nanoparticle to the water. The control of temperature is realized by rescaling velocities of copper atoms locating in the heat source. The temperature of copper rises periodically in a very short period of time to supply the heat to the liquid water.

The diameter of copper nanoparticles is $15 \AA$. At the initial stage of simulation, the liquid water region is in contact with the copper nanoparticle, which includes 51,522 atoms in the FCC unit cells. The lattice constant of liquid water is chosen as $4.9 \AA$, which agrees with the density of liquid water $1 \mathrm{~g} / \mathrm{cm} 3$. During the modeling process, an empty space is left to ensure the number of water atoms is the same for both cases. In other words, once the nanoparticle changes, its occupied space is compensated by increasing the diameter of the aforementioned space.

The Lennard-Jones (LJ) fluid is chosen to model the properties of real fluids since it has a good representation of transitions between vapor-liquid, solid-liquid, and solid-vapor phases, as well as the critical points. The interatomic interaction between copper-water and waterwater is described by the standard 12-6 LJ potential function with a cutoff distance of $10 \AA$, which has already been shown to be a simple and reliable potential function. Parameters used for atomic interactions are listed in Table 1.
Table 1. Parameters for interaction function.

\begin{tabular}{ccc}
\hline Parameters & Values & Units \\
\hline$\varepsilon_{\mathrm{OO}}$ & 0.16275 & $\mathrm{eV}$ \\
$\sigma_{\mathrm{OO}}$ & 3.16435 & $\AA$ \\
$\mathrm{q}_{\mathrm{H}}$ & 0.52 & $\mathrm{e}$ \\
$\varepsilon_{\mathrm{CuO} \text { (hydrophobic) }}$ & 0.0114 & $\mathrm{eV}$ \\
$\varepsilon_{\mathrm{CuO} \text { (hydrophilic) }}$ & 0.0684 & $\mathrm{eV}$ \\
$\sigma_{\mathrm{CuO}}$ & 2.8675 & $\AA$ \\
$\varepsilon_{\mathrm{OH}}$ & 0.0000 & $\mathrm{eV}$ \\
$\sigma_{\mathrm{OH}}$ & 0.0000 & $\AA$ \\
\hline
\end{tabular}

The embedded atom method (EAM) potentials are used in this study. It represents the total energy of the energy of the system as two additive terms, a pairwise sum of interactions between atoms, and a term representing the electron density of each atomic site

$$
U_{i}\left(r_{i j}\right)=F_{\alpha}\left(\sum_{i \neq j} \rho_{\beta}\left(r_{\mathrm{ij}}\right)+\frac{1}{2} \sum_{i \neq j} \emptyset_{\alpha \beta}\left(r_{i j}\right)\right.
$$

where rij is the separation distance between atoms, $\varnothing \alpha \beta$ is a pairwise potential function, $\alpha$ and $\beta$ are the element types of atoms $i$ and $j, \rho \beta$ is the contribution to the electron charge density from atom $j$ of type $\beta$ at the location of atom $i$, and $F \alpha$ is an embedding function that represents the energy required to place atom i of type $\alpha$ into the electron cloud.

A 4-site TIP4P model is used for the water (Fig. 2) because of its wide applicability. The bonded and nonbonded potentials used to describe the interaction between water atoms are: 


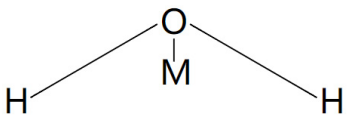

Fig. 2. Tip4p water model

$$
\begin{aligned}
& \mathrm{U}_{\text {stretch }}=K_{b}\left(b-b_{0}\right)^{2} \\
& U_{\text {bend }}=K_{\theta}\left(\theta-\theta_{0}\right)^{2} \\
& U_{\text {Van-der-Waals }}=4 \varepsilon\left[\left(\frac{\sigma}{r_{i j}}\right)^{12}-\left(\frac{\sigma}{r_{i j}}\right)^{6}\right] \\
& U_{\text {electrostatic }}=\frac{q_{i} q_{j}}{4 \pi \varepsilon_{0} r_{i j}}
\end{aligned}
$$

where $U_{\text {stretch }}$ and $U_{\text {bend }}$ are bonded potential terms that correspond to two types of atom movement, bond stretching and angle bending; $U_{\text {Van-der-Waals }}$ and $U_{\text {electrostatic }}$ are two non-bonded terms, the van der Waals interaction energy and the electrostatic interaction energy, respectively; $U_{\text {Van-der-Waals }}$ is described by a common expression, i.e., the L-J 12-6 function. Both the L-J and Columbic interactions are intermolecular interactions, which are calculated between nonbonded atoms. The interactions between $\mathrm{H} 2 \mathrm{O}$ molecules and $\mathrm{Cu}$ atoms are calculated only by considering the interactions between oxygen atoms and $\mathrm{Cu}$ atoms using the L-J 12-6 potential. In order to reduce the computational amount, a cutoff radius of $10.0 \AA$ is used for the force calculation. Table 2 lists the other parameters used for TIP4P with a long-range Columbic solver and interaction functions.

Table 2. Potential parameters of water.

\begin{tabular}{cc}
\hline Parameter & Value \\
\hline O mass & 15.9994 \\
H mass & 1.008 \\
$r_{0}$ of OH bond & 0.9572 \\
HOH angle & 104.52 \\
OM distance & 0.1250 \\
\hline
\end{tabular}

All simulations are divided into three stages using the Nose-Hoover barostat. First, the whole system is conducted under NVT ensemble $(300 \mathrm{~K})$ for a period of 100,000 time-step to reach the thermal equilibrium state.

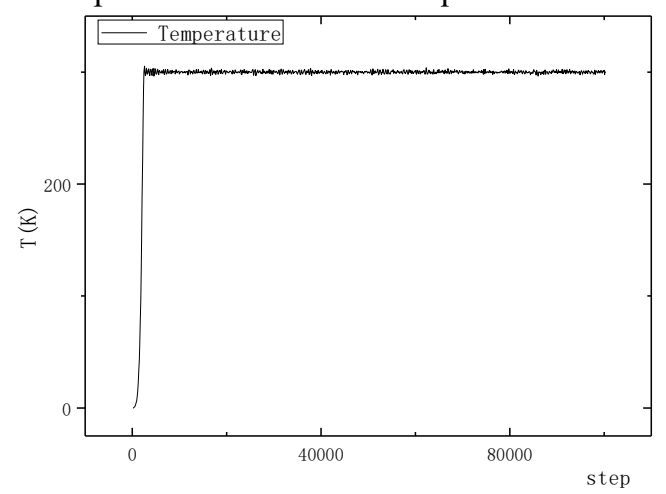

(a)

Fig. 3. (a) Temperature of the whole system in the first stage vs. the step numbers. (b) Nanoparticle and water temperatures in the first stage vs. the step numbers.
During this period, Nose-Hoover thermostat is used for copper and water separately. After the equilibration stage, the global thermostat is removed, and the whole system is conducted under NVE for 10,000 time-steps to keep the system equilibrium for a period of time (the second stage). At the third stage, a heat source is applied. The nanoparticle is then heated by rescaling the velocities of copper atoms at every time step with a constant heat intensity. The copper nanoparticle is not allowed to freely diffuse by neglecting the effect of the Brownian motion of particle. During the transition period, the temperature of water increases and the density of the water changes. The temperature and the density of different regions are different.

The region of water is divided into seven shells, starting from the outer surface of the nanoparticle (in a spherical area of $15 \AA 2$ ) to $50 \AA$ in radius. It means on can divide the area of the sphere shell every $5 \AA$ into layers. In order to better distinguish these stratified areas of water, we use the inner and outer radius of the spherical shell to represent the area of water.

\section{Results and analysis}

The melting point of copper nanoparticle depends on its size. The early melting will occur at a lower temperature due to the increasing specific surface area, although the melting temperature of copper is $1357.77 \mathrm{~K}$. To avoid the melting of copper nanoparticle and also as a proof of the reliability of the MD simulation, a separated MD study of the melting of individual copper nanoparticle is conducted. For the $3 \mathrm{~nm}$ particles containing 1,207 atoms used in this simulation, the melting point was found to be above 800 $\mathrm{K}$. Therefore, the temperature of the nanoparticle is limited no more than $600 \mathrm{~K}$.

During stages I and II, the average temperature of the system reaches a steady state at about $10,000 \mathrm{fs}$, as shown in Fig. 3. The four parameters of the system, i.e., temperature, density, total energy and total potential energy, are basically stable. During stage III, the copper temperature rises rapidly from $300 \mathrm{~K}$ to $450 \mathrm{~K}$ in a very short period of time (about $100 \mathrm{ps).} \mathrm{Due} \mathrm{to} \mathrm{the} \mathrm{control} \mathrm{of}$ atom velocity, the rising speed of copper temperature is very stable, as can be seen in Fig. 4.

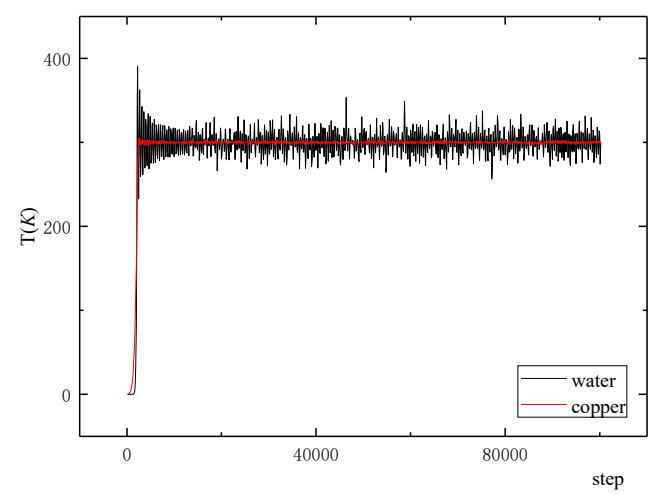

(b) 


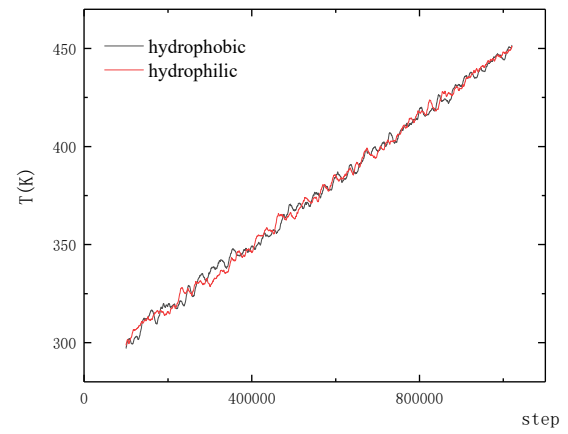

Fig. 4. Temperature of copper during the third stage vs. the step numbers.

Heat is transferred from the copper nanoparticle to the water pool. The increasing rates of water temperature for both hydrophobic hydrophilic conditions are apparently different. Figure 5 shows that the temperature of the water near the hydrophilic nanoparticles rises faster than near the hydrophobic one. As compared to the hydrophobic surface, higher temperature can be observed for almost all the time steps during the simulation and hence higher heat flux is expected at the interface between water and copper. This indicates that a hydrophilic surface can reduce the heat transfer resistance during rapid boiling of water.

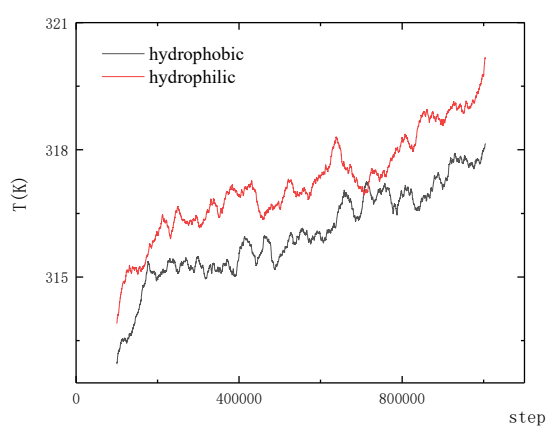

Fig. 5. Temperature of the whole system in the third stage vs. the step numbers.

During the third stage, the temperature of each layer of water is averaged for the two surfaces. Figure 6 shows an apparently higher temperature gradient in the nanometer region with a radius of $15 \AA$ to $50 \AA$ and thus heat can be transferred faster for the hydrophilic surface than for the hydrophobic one.

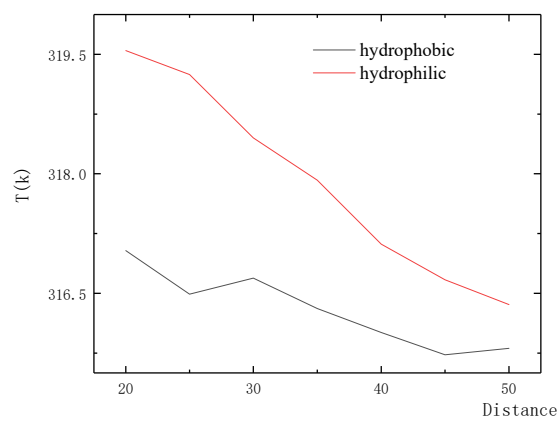

Fig. 6. Water temperatures in the first stage vs. the distance.
Figure 7 shows the density of water at the surface of hydrophilic nanoparticles is greater at the beginning of the third stage. The density difference is more significant at a distance of $2 \mathrm{~nm}$ from the surface of the nanoparticle.

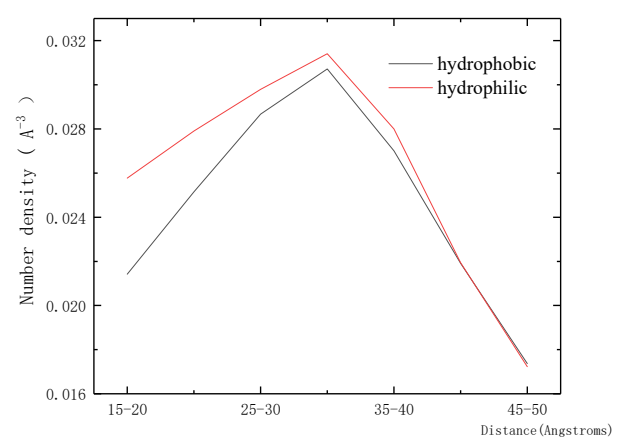

Fig. 7. Water density at 160,000 steps.

The changes of the water density of each layer of water outside the hydrophilic and hydrophobic nanoparticles with respective to the number of steps can then be analyzed. As shown in Figs. 8 and 9, for both hydrophilic and hydrophobic cases, the density of interior water tends to decrease while the density of water which is far from the nanoparticle increases. However, the density of interior water decrease more rapidly and significantly for the hydrophilic surface.

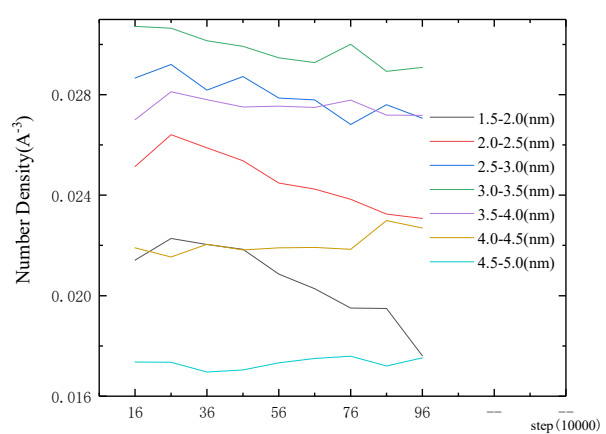

Fig. 8. Number density of water around hydrophobic particle in the third stage vs. step numbers.

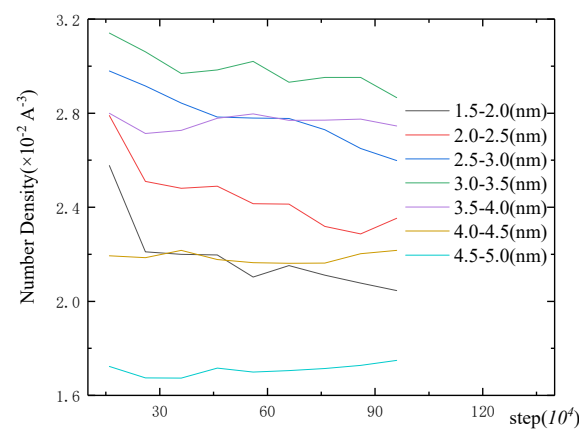

Figure 9. Number density of water around hydrophilic particle in the third stage vs. the step numbers.

In order to illustrate more clearly the density variation of water in the vicinity of the nanoparticle surface, the 
density changes in the annular regions with radius of 15 $20 \AA$ and of $45-50 \AA$ are given in Figs. 10 and 11 , respectively. The abovementioned investigation reveals the tendency of outward diffusion for the interior water molecules. It also reveals that the interfacial thermal resistance between the water and the nanoparticle is smaller when the surface is hydrophilic. This can be attributed to the fact that more water molecules emerge at the hydrophilic surface than at the hydrophobic surface, and the increased number density of water molecules near the surface results in enhanced heat transfer between the copper nanoparticle and the water molecules since more energy is transported as the atom distance decreases. Since the simulation time is limited in $100 \mathrm{ps,} \mathrm{more} \mathrm{accurate}$ results require that the simulation time to be larger than 1,000 ps.

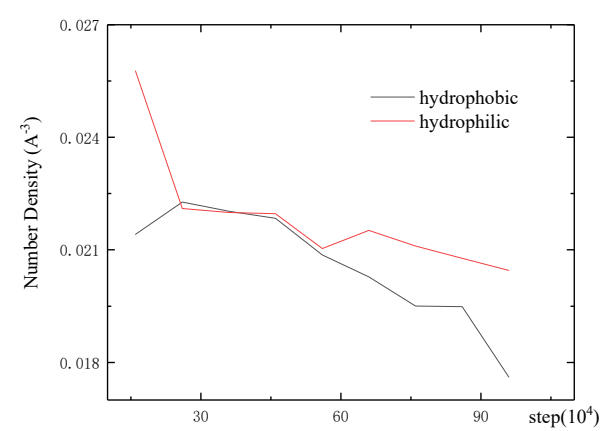

Figure 10. Comparison of the density changes with time step in both wetting conditions for 15-20 ^ layers.

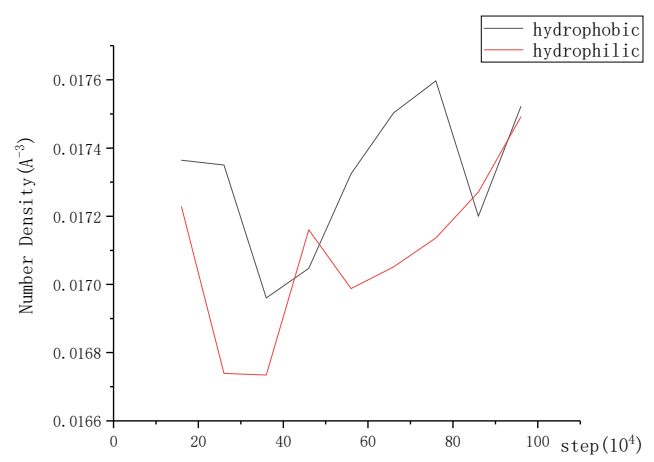

Figure 11. Comparison of density changes with time step in both wetting conditions for 45-50 ̊̊ layers.

\section{Conclusions}

Molecular dynamics simulation of heat transfer from a nanoparticle to the surrounding liquid pool is carried out to study the effect of solid-liquid interfacial wettability on the boiling phenomena. The interactions among copper atoms are described by the embedded atom method (EAM) potentials, and the TIP4P water model is used to describe water atoms. The results show that the interfacial thermal conductance is influenced by the interfacial wettability.
Increasing the wettability between the particle and the fluid reduces the interfacial resistance. The hydrophilic surface can effectively reduce the thermal resistance between water and copper nanoparticles.

\section{References}

1. US Choi. Enhancing Thermal Conductivity of Fluids with Nanoparticles. Developments and Applications of Non-Newtonian Flows. ASME, NY, 1995: 99-105.

2. Nan Wang, Jun Chen, Qingsong An, et al. Molecular dynamics study of the dispersion stability of nanofluids . Journal of Engineering Thermophysics, 2011, 32(7):1107-1110. (In Chinese)

3. Zakaria I A, Wan A N W M, Mamat A M I, et al. Thermal performance of Al2O3in water - ethylene glycol nanofluid mixture as cooling medium in mini channel. AIP Publishing LLC, 2015:2494-2507.

4. Li X, Zhu D, Wang X. Evaluation on dispersion behavior of the aqueous copper nano-suspensions. Journal of Colloid \& Interface Science, 2007, 310(2):456-63.

5. Min Zhang, Michael Siedow, Gregory Saia, et al. Molecular Scale Aspects of Liquid Contact on a Solid Surface. Solid Surface Therm. Sci. Eng, 2002, 70(8):e458.

6. Maruyama S, Matsumoto S, Shoji M, et al. A Molecular Dynamics Study Of Interface Phenomena Of A Liquid Droplet. Begel House Inc., 1999.

7. Kimura T, Maruyama S. Molecular Dynamics Simulation of Water Droplet in contact with a Platinum Surface. 2008: 537-542.

8. Maruyama S, Kimura T. A Molecular Dynamics Simulation of Bubble Nucleation on Solid Surface. Nihon Kikai Gakkai Ronbunshu B Hen/transactions of the Japan Society of Mechanical Engineers Part B, 2000, 65(638):3461-3467.

9. Ji C Y, Yan Y Y. A molecular dynamics simulation of liquid-vapour-solid system near triple-phase contact line of flow boiling in a microchannel. Applied Thermal Engineering, 2008, 28(2-3):195202.

10. Carey V P, Wemhoff A P. Thermodynamic analysis of near-wall effects on phase stability and homogeneous nucleation during rapid surface heating. International Journal of Heat \& Mass Transfer, 2005, 48(25):5431-5445.

11. Wang C S, Chen J S, Shiomi J, et al. A study on the thermal resistance over solid-liquid-vapor interfaces in a finite-space by a molecular dynamics method. International Journal of Thermal Sciences, 2007, 46(12):1203-1210.

12. Xue L, Keblinski P, Phillpot S R, et al. Two regimes of thermal resistance at a liquid-solid interface. Journal of Chemical Physics, 2003, 118(1):337-339. 
13. S. Plimpton, Fast parallel algorithms for short-range molecular dynamics. J. Comput. Phys., 1995, 117: 142. 